\title{
SOLIDARIDADES EN CONFRONTACIÓN, RESISTENCIAS Y ÓRDENES SOCIALES EN TIEMPOS DE PANDEMIA ${ }^{1}$
}

Clashing solidarities, resistances, and social orders in times of pandemic

\author{
CASTRO, José Esteban²
}

\begin{abstract}
Resumen: La pandemia de COVID-19 encontró América Latina en un contexto político muy desfavorable para los sectores populares y las comunidades históricamente marginalizadas, especialmente afrodescendientes e indígenas. Con pocas excepciones, se verifica una expansión de políticas regresivas, autoritarias e incluso criminales en muchos de estos países, que han debilitado o incluso desmantelado los limitados sistemas de solidaridad social institucionalizados. El artículo discute la noción de "solidaridades en confrontación" y pone énfasis en los elementos que dan cohesión a los grupos de poder responsables por estos procesos, particularmente la persistencia del darwinismo social y de diferentes formas de discriminación que sustentan la justificación y naturalización de las desigualdades estructurales. El trabajo examina ejemplos de estas formas de solidaridad al interior de los grupos dominantes, su expresión concreta en los planos discursivo y político, y su confrontación desde espacios de solidaridad alternativos, centrados en la defensa de la vida y en la lucha por la emergencia de órdenes sociales más justos y substantivamente democráticos.
\end{abstract}

Palabras clave: Autoritarismo. Darwinismo social. Desigualdad estructural. Neocolonialismo. Solidaridades

\begin{abstract}
The COVID-19 pandemic arrived in Latin America in a political environment very hostile for the popular sectors and the communities historically marginalized, particularly Afrodescendent and Indigenous communities. With few exceptions, there is an expansion of regressive, authoritarian, even criminal policies in many of these countries, which have weakened or even dismantled the limited systems of institutionalized social solidarity. The article discusses the notion of "clashing solidarities" and places the emphasis on the elements that give cohesion to the power groups responsible for these processes, particularly the persistence of social Darwinism and different forms of discrimination that provide support for the justification and naturalization of structural inequalities. The work examines examples of the forms of solidarity within the dominant groups, their concrete expressions in the discursive and political domains, and how these are confronted from alternative spaces of solidarity centred on the defence of life and the struggle for more just and substantively democratic social orders.
\end{abstract}

Keywords: Authoritarianism. Social Darwinism. Structural inequality. Neocolonialism. Solidarities.

\footnotetext{
${ }^{1}$ Recebido em: 02 Set. 2020 | 30 Set. 2020.

2 Investigador Principal, Consejo Nacional de Investigaciones Científicas y Técnicas (CONICET), Universidad Nacional de General Sarmiento (UNGS), Emeritus Professor, Newcastle University, Reino Unido.
} 


\section{Introducción}

Los pocos meses transcurridos desde el inicio de la pandemia de COVID-19 en marzo de 2020 hasta la fecha de escribir este trabajo a fines de agosto del mismo año, período que se nos ha presentado como una interminable pesadilla, pueden verse como un inesperado laboratorio para revisitar la relación entre la formación de lazos sociales de solidaridad y su relación con los procesos de consolidación, ruptura y emergencia de órdenes sociales. Los impactos de fenómenos como las epidemias, pandemias y otros desastres denuncian la fragilidad y vulnerabilidad de los sistemas humanos de contención y resolución de las amenazas que confronta la especie y la vida en el planeta más generalmente. El carácter antropogénico de muchos de los procesos y factores que intervienen en la producción de dichas amenazas y, frecuentemente, en su concreción material, pone en cuestión nuestra capacidad de reducir los niveles de fragilidad y vulnerabilidad que quedan expuestos recurrentemente por la devastación causada por estos fenómenos. El carácter en gran medida sociogenético de estos y otros fenómenos destructivos añade grados de incertidumbre con respecto a la capacidad humana para enfrentar y resolver favorablemente estas confrontaciones. Sin embargo, además de poner a prueba nuestras capacidades, la ocurrencia de estos procesos también nos lleva a cuestionar el grado de determinación que existe en las comunidades humanas, y especialmente en sus clases dominantes, para asegurar que la resolución favorable de dichas confrontaciones, en caso de lograrse, tenga alcance universal. Cuestiona el significado y la misma posibilidad de la solidaridad social, entendida como solidaridad a nivel de la especie.

En relación con esto, el artículo examina ejemplos de algunas contradicciones extremas que se han manifestado en América Latina a partir de la crisis desatada por la pandemia, particularmente entre los intentos de revitalizar las políticas de "solidaridad institucionalizada" centradas en el rol del Estado para proteger a la población y el ataque virulento contra dichos intentos por parte de sectores sociales cohesionados por el darwinismo social y el ultraliberalismo. La primera sección adopta una perspectiva histórica para discutir el carácter social de la "solidaridad" como proceso contradictorio, como confrontación de "solidaridades" construidas en torno a intereses materiales, valores, creencias y determinaciones rivales y frecuentemente antagónicas. La segunda 
sección aborda el problema de las desigualdades estructurales en América Latina, con énfasis en la situación de las comunidades indígenas y afrodescendientes, y discute el atrincheramiento de sectores dominantes de las elites intelectuales, políticas y empresariales de la región en torno a la defensa de visiones clasistas, colonialistas, discriminatorias y racistas con profundas consecuencias negativas para los sectores sociales marginalizados y excluidos y para el propio futuro de los procesos de democratización substantiva de nuestras sociedades. La sección final hace una breve referencia a formas solidarias alternativas, algunas de ellas ancestrales, que constituyen formas de resistencia a la opresión que se ve revitalizada por los procesos de recolonización y reimperialización que experimenta América Latina, cuyos impactos se ven agravados como consecuencia de la pandemia. Esas formas también constituyen potencialmente uno de los fundamentos para el desarrollo de formas de solidaridad a nivel de la especie, que puedan dinamizar la emergencia de órdenes sociales superadores.

\section{La “solidaridad” en cuestión: solidaridades y órdenes sociales}

El concepto sociológico de "solidaridad", entendido como un proceso neutro, como planteó James Mark Baldwin hace más de un siglo, se refiere a "un asunto de relaciones mutuas de un grupo de individuos unos con otros" (Baldwin, 1910: 817) ${ }^{3}$. El propio Baldwin propuso en su breve ensayo que para poder hablar de formas de organización humana "verdaderamente sociales" se requiere el desarrollo de formas de "solidaridad de inteligencia, de convicción, de sentimiento elevado, que reemplacen a la solidaridad del mero instinto o del sentimiento ciego [...] se trata de una solidaridad resultante de la intención consciente y de la cooperación voluntaria" (Id.: 825-6). Otro Baldwin, Peter, escribiendo a inicios de la década de 1990 sobre "la política de la solidaridad social", analizó retrospectivamente el desarrollo del Estado de Bienestar en Europa como la forma más cercana a lo que podría llamarse "solidaridad institucionalizada", explorando la diversidad de procesos sociales que de una $u$ otra forma, sirviendo intereses desemejantes y contradictorios, de algún modo produjeron el fenómeno de crear formas institucionalizadas de redistribución de la riqueza, es decir formas institucionalizadas de

\footnotetext{
${ }^{3}$ Salvo que se indique lo contrario, las traducciones al español son del autor.
} 
solidaridad social (Baldwin, 1990: 10-11, 29). Observando estos procesos desde nuestro momento histórico, a inicios de la tercera década del siglo veintiuno y en medio de una pandemia en gran medida sin precedentes, queda patente la fragilidad, transitoriedad y finitud de dichas formas de solidaridad institucionalizada, las cuales, por otra parte, y con pocas excepciones, ni siquiera alcanzaron niveles de desarrollo significativos afuera del núcleo europeo. También nos lleva a reflexionar sobre los procesos históricos de desintegración y reformulación de los lazos de solidaridad social y repensar lo que podemos denominar las "contradicciones de la solidaridad", el hecho de que aquello que Baldwin a inicios del siglo veinte denominaba la "solidaridad de inteligencia, de convicción, de sentimiento elevado" conectadas con la intencionalidad consciente y la cooperación voluntaria (Baldwin, 1910) no resulta de procesos progresivos, lineales o necesariamente universalistas. Como parte de los procesos de formación histórica de órdenes sociales, diversas formas de "solidaridad" emergen a partir de entramados cambiantes de relaciones humanas en flujo, para utilizar una frase de Norbert Elías sobre los procesos de transformación social, su direccionalidad y, particularmente, las complejas configuraciones sociales emergentes de los mismos (Elias, 1994). En función de las relaciones entre formas de solidaridad y órdenes sociales en el marco de esta pandemia, por ejemplo, es fundamental considerar las formas de "solidaridad institucionalizada" que emergieron en algunas zonas de Europa durante la segunda mitad del siglo veinte. Dejando de lado por un momento las controversias sobre esas formas de "solidaridad institucionalizada" encarnadas en los sistemas de seguridad social en manos de los Estados-Nación y las críticas al carácter capitalista de tales sistemas y sus graves implicaciones, estos todavía representan las únicas formas existentes de protección contra estas calamidades de que disponen amplios sectores de la población mundial que están excluidos del acceso privado, mercantil a dicha protección. Es necesario destacar el enorme esfuerzo histórico, en gran medida exitoso, realizado por fracciones de los sectores dominantes a nivel global para limitar, reducir e incluso desmantelar dichas formas de solidaridad social institucionalizada o impedir el desarrollo de formas similares en aquellos territorios en los que dichas formas nunca consiguieron arraigarse o siquiera introducirse. Dichos procesos de desmantelamiento y ataque a los sistemas de solidaridad institucionalizada han reducido significativamente la capacidad de reacción colectiva a estas amenazas, literalmente "desarmando" a amplios sectores de la población y 
reduciéndolos a un estado de indefensión, negándoles, en última instancia, el derecho a existir.

En relación con lo anterior, un aspecto fundamental que debe ser destacado es la persistencia eficaz del darwinismo social, incluso en sus formas extremas asociadas con el liberalismo de mercado a ultranza pero que también permean el pensamiento de otros sectores menos regresivos del espectro político. En el contexto actual, al leer y escuchar los posicionamientos de diversos sectores sobre cuál debe ser la respuesta a la pandemia, es posible, literalmente, retrotraerse a mediados del siglo diecinueve, cuando se postulaban los principios del darwinismo social en el marco de las graves epidemias que arrasaban particularmente a los barrios proletarios y destituidos de las ciudades europeas. Como lo planteó Eric Hobsbawm en su descripción histórica de la situación,

[1]os poblados y las áreas industriales crecieron rápidamente, sin plan ni supervisión, y los servicios más elementales de la vida urbana fracasaron absolutamente en seguir el ritmo de crecimiento: limpieza de las calles, servicio de agua, saneamiento, ni que hablar de viviendas para la clase trabajadora. La consecuencia más obvia de este deterioro urbano fue la reaparición de epidemias masivas de enfermedades (principalmente de origen hídrico) contagiosas, notablemente el cólera, que reconquistó Europa a partir de 1831 y barrió el continente de Marsella a San Petersburgo en 1832 y nuevamente después. [...] El desarrollo urbano de este período fue un proceso gigantesco de segregación de clase, que empujó a los nuevos sectores de trabajadores pobres a grandes lodazales de miseria ubicados fuera de los centros de gobierno y negocios y de las nuevas áreas residenciales de la burguesía (Hobsbawm, 1975: 203).

Si eliminamos las referencias geográficas y cronológicas del relato, un lector actual podría tranquilamente comentar que la descripción corresponde a muchas ciudades latinoamericanas contemporáneas. En relación con esto, vale la pena recordar la intervención de Herbert Spencer en oposición a la aprobación por parte del Parlamento Británico de una actualización de las "Leyes de Pobres" [Poor Laws], un antecedente temprano de las formas de "solidaridad institucionalizada" que emergerían a mediados del siglo veinte. En su libro "Estática Social, o las Condiciones Esenciales para la Felicidad Humana”, publicado en 1850, Spencer argumentó que

[e]l bienestar de la humanidad existente y su desarrollo hacia [el estado] de última perfección, son asegurados por la misma disciplina benevolente, pero severa, a la que se encuentra sujeta el resto de la creación: una disciplina despiadada en la producción del bien: una ley orientada a perseguir la felicidad que nunca se desvía de su curso para evitar sufrimientos parciales y temporarios [....] esos desplazamientos de los débiles a los codazos por parte de los fuertes, que dejan a tantos "en 
sombras y miserias", son los decretos de una benevolencia mayor y de larga visión. [...] en conexión con los intereses de la humanidad universal, esas duras fatalidades pueden verse como estando plenas de la beneficencia más elevada -la misma beneficencia que lleva temprano a sus tumbas a los niños de padres fallecidos y separa a los de bajo espíritu, a los intemperados y a los debilitados como a las víctimas de una epidemia [...]. Debemos llamar filántropos espurios a quienes, para prevenir la miseria presente, impondrán una miseria aún mayor a las generaciones futuras. Todos los defensores de una ley de pobres deben, por lo tanto, ser clasificados como tales. [...] Ciegos al hecho que, de acuerdo con el orden natural de las cosas, la sociedad constantemente excreta a sus miembros enfermizos, imbéciles, lentos, vacilantes, desleales, estos hombres irreflexivos, aunque bien intencionados, abogan por una interferencia que no solamente detiene el proceso purificador sino que además incrementa la decadencia (Spencer, [1850] 1883: 353355).

Decir que con estas referencias estamos retrocediendo al siglo diecinueve sería inconvenientemente diplomático. Las corrientes retrógradas del siglo diecinueve están activas entre nosotros, con gran vitalidad y controlan importantes mecanismos de poder a nivel global. En cierto modo, los argumentos de Spencer en la década de 1850 oponiéndose a la aprobación de leyes para proteger a los trabajadores y a los sectores marginalizados son más sofisticados que los igualmente repudiables argumentos de los darwinistas sociales del siglo veintiuno, aunque causen reacciones similares. Es fácil encontrar ejemplos de estos posicionamientos en los representantes contemporáneos de las derechas simultáneamente ultraliberales y ultraconservadoras, lo cual hacemos en la siguiente sección. Pero lo que debe preocupar es la vitalidad de los principios del darwinismo social en sectores que se identifican con el espectro de fuerzas sociales "progresistas", que critican las políticas públicas de "solidaridad institucionalizada" implementadas por algunos países para enfrentar la pandemia con argumentos no muy diferentes a los de Spencer.

Centrándonos en América Latina, el debate sobre las relaciones entre formas de "solidaridad" y órdenes sociales nos conduce necesariamente al examen de estos procesos en el marco de los procesos de (re)colonización y (re)imperialización externa e interna (González Casanova, 1963: 300). En esta región, dado el carácter histórico de sus profundas desigualdades estructurales, se facilita el hacer observable las contradicciones de la "solidaridad" como mecanismo de diferenciación social, como mecanismo de inclusión-exclusión, de ordenación social. En América Latina es posible desromantizar la 
noción de solidaridad recuperando el carácter sociológico del concepto para hablar de "solidaridades en confrontación", solidaridades que responden a proyectos rivales y hasta antagónicos de la especie humana, que también asumen formas contradictorias en el marco de diversas desigualdades estructurales y estructurantes, de clase, de género, étnicas, etarias, etc., crudamente expuestas por la actual pandemia. También debe hablarse de solidaridades dominantes y opresivas, como la solidaridad que cohesiona a importantes sectores de la población en torno a los principios del darwinismo social y a la práctica de diversas formas de discriminación y que defienden a la desigualdad estructural como una función positiva y necesaria, que garantiza el orden social. El grado de profundización de estas contradicciones puede ilustrarse con el extremo al que parecen estar siendo puestas a prueba las solidaridades religiosas, por ejemplo en el caso de los grupos cristianos evangélicos que en Brasil parecen haber reemplazado su compromiso, al menos formal, con los preceptos de la solidaridad predicada por su líder espiritual y han adoptado lazos de solidaridad construidos en torno a un programa político negador del derecho a la protección pública e impulsor de prácticas genocidas, como el que encarna el actual Presidente Jair Bolsonaro (2019-), aunque el fenómeno se registra también en los Estados Unidos y en gran parte de América Latina (Oualalou, 2019; Correia y Dip, 2020; Lissardy, 2020).

Con el objeto de mantener este texto dentro de los límites de un breve artículo, me centraré en la discusión de algunos aspectos relevantes a la situación de las comunidades indígenas y afrodescendientes, que históricamente y hasta el presente han estado expuestas a grados extremos de marginalización y exclusión, como repetidamente lo confirma una evidencia empírica abrumadora (CEPAL y ACNUDH, 2020; CEPAL y FILAC, 2020). Como lo denuncian una serie de recientes informes de instituciones de las Naciones Unidas, la pandemia está afectando de manera desproporcionada a los pueblos indígenas y afrodescendientes (OHCHR, 2020; OPS, 2020), lo cual es producto de las desigualdades estructurales y de la discriminación de que son objeto, y al mismo tiempo contribuye a la reproducción y profundización de dichas desigualdades y formas de discriminación. Pero es aún más grave constatar el negacionismo clasista, racista y neocolonialista de las fracciones sociales dominantes en muchos países de la región, que continúan justificando los genocidios históricos y la exclusión y marginalización presente de estas comunidades, como discutimos a continuación. 


\section{Solidaridad como proceso de inclusión-exclusión: su observabilidad en tiempos de pandemia}

En un Manifiesto difundido en abril de 2020 por la Fundación Internacional para la Libertad (FIL), organización ultraliberal con fuertes tonalidades derechistas presidida por el Premio Nobel de Literatura Mario Vargas Llosa, titulado "Que la pandemia no sea un pretexto para el autoritarismo", los firmantes expresaron su "solidaridad a las familias enlutadas" por la pandemia (FIL, 2020). En la primera línea de firmas aparecen los nombres de varios expresidentes iberoamericanos, entre los cuales, dado lo breve de este artículo, quiero destacar a tres por su relevancia para esta discusión: Julio María Sanguinetti (Uruguay), Álvaro Uribe Vélez (Colombia) y Mauricio Macri (Argentina), entre otros nombres que llevan a cuestionar el significado de los conceptos de "libertad", "solidaridad" y autoritarismo" a los que se refiere el Manifiesto. Tomemos por ejemplo el caso del ex Presidente de Uruguay, Julio María Sanguinetti (1985-1990 y 1995-2000). En 2009 Sanguinetti fue objeto de severas críticas en su país por publicar un artículo de prensa en el que incluyó declaraciones que fueron denunciadas como colonialistas y racistas, denigrantes para la etnia Charrúa y para las comunidades indígenas uruguayas en general. Entre otros temas, argumentó que

No hemos heredado de ese pueblo primitivo ni una palabra de su precario idioma, ni el nombre de un poblado o una región, ni aun un recuerdo benévolo de nuestros mayores, españoles, criollos, jesuitas o militares, que invariablemente les describieron como sus enemigos, en un choque que duró más de dos siglos y les enfrentó a la sociedad hispano-criolla que sacrificadamente intentaba asentar familias y modos de producción, para incorporarse a la civilización occidental a la que pertenecemos. [... el de los charrúas fue un] modo de vida que estuvo condenado desde el primer día en que se afincó la civilización española en nuestras tierras (Sanguinetti, 2009).

Este artículo, publicado en abril de 2009, intentaba intervenir en un debate nacional sobre la discriminación histórica sufrida por la etnia Charrúa, en un contexto de creciente activismo por parte de los descendientes de las comunidades indígenas y el liderazgo asumido por la Institución Nacional de Derechos Humanos y Defensoría del Pueblo (INDDHH). Pocos meses después de la publicación del artículo de Sanguinetti se 
aprobó una ley nacional, en septiembre de 2009, la cual declaró el "Día de la Nación Charrúa y de la Identidad Indígena", que desde entonces se celebra el día 11 de abril de cada año (Poder Legislativo, 2009). La ley obedece al objetivo de modificar en Uruguay una cultura dominante de negación del aporte, y hasta de la misma existencia, de las comunidades indígenas en el país. El artículo de Sanguinetti reflejaba su alineamiento con la posición que niega los crímenes cometidos contra las comunidades indígenas durante el proceso de colonización y la posterior formación del Estado-Nación y la discriminación a la que han sido sometidos los descendientes históricamente.

El episodio de 2009 no fue un hecho fortuito, una mera disquisición intelectual sobre la interpretación de registros históricos, como el texto de Sanguinetti parecería sugerir. El debate continuó y en 2016 el ex Presidente lo reavivó con un nuevo ataque a los intentos de reivindicación de las comunidades indígenas del país, con un artículo titulado "Charruismo estatal", en el que cuestiona a la INDDHH por patrocinar un evento organizado por lo que denomina "las delirantes organizaciones charruístas, que han inventado una civilización inexistente" (Sanguinetti, 2016). Representantes de la etnia Charrúa le respondieron duramente y afirmaron que ya "no estamos en la época del darwinismo social sino en la época de las reemergencias indígenas" (Delgado Cultelli, 2016). Más recientemente, ya en plena pandemia de COVID-19, el 9 de junio de 2020, pocas semanas después de firmar el Manifiesto contra el uso de la pandemia como "pretexto para el autoritarismo", Sanguinetti retomó el tema con un video en el que reafirma la superioridad de la civilización europea colonizadora y naturaliza la noción de que la existencia de etnias indígenas en el país, particularmente la Charrúa, sería un mito construido (Sanguinetti, 2020). Este video nuevamente desató fuertes reacciones por parte de las organizaciones indígenas, como la Asociación de Descendientes de la Nación Charrúa y Consejo de la Nación Charrúa: "repudiamos las expresiones del legislador Sanguinetti porque no sólo denotan discriminación y racismo hacia nuestras organizaciones, sino hacia un Pueblo Originario que luchó y entregó hasta su última gota de sangre por la libertad de este suelo" (ADENCH y CONACHA, 2020). Como afirmó Correa García en un breve análisis del video de Sanguinetti, el mismo buscaba reafirmar la visión todavía dominante en el país de que el exterminio de los indígenas fue "una desaparición lamentable pero necesaria para la consolidación en base a la igualdad, la civilización, la democracia y el progreso" (Correa García, 2020). Sanguinetti representa la reacción a lo 
que Gustavo Verdesio describió afirmando que “[u]n fantasma recorre el Uruguay: la reemergencia charrúa en un 'país sin indios'” es decir, un país en el que el "éxito del discurso que declara la extinción de los indígenas [...] es casi total” (Verdesio, 2014: 88). En este sentido, cabe remarcar que, hasta la fecha de escribirse este artículo, Uruguay, junto con Surinam y las Guayanas, continúa, siendo uno de los pocos países sudamericanos que no han ratificado el "Convenio 169 sobre pueblos indígenas y tribales en países independientes" de la Organización Internacional del Trabajo (OIT) (OIT, 1989).

Es importante destacar este caso de Uruguay, ya que la prevalencia de la visión racista, colonial y discriminatoria en este país es mucho menos conocida que, por ejemplo, en Argentina o Chile, con los cuales comparte la descripción de "países de colonos" o, como los denominó Alfred Crosby, "Neo-Europas” (Crosby 1988), aunque dicha visión también está presente en otros países y en las "Neo-Europas de otras latitudes. Sin embargo, obviamente el de Sanguinetti no es el único caso para destacar entre los firmantes del Manifiesto contra "el autoritarismo" de la Fundación Internacional para la Libertad. Es mucho más preocupante el caso de Álvaro Uribe Vélez, ex Presidente de Colombia (20022010), reelecto Senador en 2014, cargo al que renunció el 18 de agosto de 2020 mientras cumplía arresto domiciliario bajo orden de la Corte Suprema de Justicia de Colombia "por 'riesgos de obstrucción a la justicia'” en un juicio en el que es acusado de serios delitos (BBC News, 2020). Desde inicios de la década de 1980 Uribe ha ocupado diversos cargos públicos y ha acumulado una larga serie de denuncias por autoritarismo y crímenes contra la humanidad. En palabras de la organización norteamericana Oficina para América Latina en Washington (Washington Office on Latin America - WOLA) comentando el juicio que lleva a delante la Corte Suprema de Justicia,

Uribe siempre ha estado a un grado de distancia de crímenes contra la humanidad y de conductas exentas de rendición de cuentas [unaccountable] y autoritarias. [...] Esperamos que [este juicio] inicie el proceso de remover el chaleco de teflón de Uribe para que todas las víctimas de esos crímenes finalmente puedan tener justicia" (WOLA, 2020; ver también: Pachico, Sánchez-Garzoli et al., 2019).

Ciertamente, la historia de Uribe en este aspecto es muy larga. En enero de 2009, el ex Presidente de Estados Unidos Georg W. Bush (2001-2009), de quien Uribe fue un aliado clave, le otorgó al entonces Presidente de Colombia la Medalla de la Libertad, provocando numerosas reacciones. Entre otras, Lori Wallach, Directora entonces de la División de 
Vigilancia del Comercio Global de la organización Ciudadano Público (Public Citizen's Global Trade Watch Division), basada en Washington DC, denunció que

El otorgamiento por parte del Presidente Bush de la Medalla de la Libertad al Presidente colombiano Álvaro Uribe es indignante y ofensivo dados los horríficos abusos a los derechos humanos de la administración de Uribe, como lo documentan los miles de líderes sindicales, afrocolombianos e indígenas asesinados, atacados o desplazados y los vínculos de la administración con asesinos paramilitares de derecha. ¿Cómo es posible que Bush otorgue nuestro máximo honor civil al líder de un país con estatus de paria mundial debido a su aplastamiento sistemático de los derechos humanos más básicos? (Wallach, 2009).

No me detengo aquí en los pormenores de por qué el ex Presidente Bush le otorgó esa distinción, a lo cual deberíamos agregar cuáles son las razones para que el 15 de agosto de 2020 el Vicepresidente de Estados Unidos Mike Pence (2017-) demandase la inmediata liberación de Uribe de su arresto domiciliario, afirmando

nos unimos a todas las voces amantes de la libertad alrededor del mundo en solicitar a los oficiales colombianos que dejen a este Héroe, quien recibió la Medalla Presidencial de la Libertad otorgada por los Estados Unidos, para que pueda defenderse como un hombre libre (Reuters, 2020).

Este apoyo del gobierno de Estados Unidos a Uribe amerita un análisis que excede los objetivos y alcance de este breve trabajo. Pero debo destacar aquí dos puntos directamente relacionados con el tema de esta sección: el carácter racista, autoritario, discriminatorio y colonialista de las intervenciones públicas y las prácticas de Uribe. Por ejemplo, en declaraciones a la televisión colombiana durante su período como Senador, en octubre de 2016, Uribe declaró “[e]ste no es un país de tribus africanas, es un país de instituciones". Esa declaración le valió una fuerte respuesta por parte de los colectivos afrocolombianos del país, quienes declararon que "enunciados racistas como el que manifiesta el expresidente Álvaro Uribe Vélez marcan las fronteras de desintegración que han mantenido excluidas a las poblaciones afrocolombianas de los procesos de construcción del Estado" (Amira, 2016, negritas en el original).

Otro ejemplo corresponde a un evento más reciente, que se produjo en abril de 2019, cuando en su cargo de Senador, desde el que ejerce amplia influencia sobre el Presidente Iván Duque Márquez (2018-), Uribe realizó fuertes declaraciones públicas contra la decisión del gobierno de aceptar algunos puntos de negociación en respuesta a las demandas presentadas por las comunidades indígenas del suroeste del país durante 
medidas de protesta masiva organizadas en la forma de una "Minga ${ }^{4}$ por la defensa de la vida, el territorio, la democracia, la justicia y la paz". Aunque luego intentó desmentir el sentido de su mensaje, Uribe aparentemente justificó la posibilidad de una masacre de los indígenas movilizados. En un video que hizo público, Uribe argumentó que los indígenas estaban infiltrados por grupos armados y que, por lo tanto, "si la autoridad, serena, firme y con criterio social implica una masacre es porque del otro lado hay violencia y terror más que protesta" (Noticias Uno Colombia, 2019). Las comunidades indígenas desmintieron las acusaciones de infiltración y denunciaron que con sus palabras Uribe estaba "promoviendo abiertamente el odio y el asesinato" (PARES, 2019) y "justificando la no atención a los sectores sociales marginados y olvidados de este país [... exponiéndolos a] una masacre anunciada" (CRIC, 2019). Si bien la masacre no se produjo en ese momento, la matanza sistemática de líderes sociales, incluyendo líderes indígenas y afrodescendientes continúa sin descanso en la región y en Colombia al momento de escribirse este artículo (Pardo, 2020). Como lo describe otro informe publicado durante la preparación de este artículo, el país continúa sufriendo "la otra pandemia", ya que "[e]l [corona]virus no ha detenido el asesinato sistemático de líderes sociales" (Medina Gallego, 2020). En contraste, cabe destacar que las comunidades indígenas del Cauca han estado organizando mingas solidarias en el contexto de la pandemia, incluyendo algunas para distribuir alimentos en las ciudades donde habita la población blanca y mestiza (CRIC, 2020), lo cual comentamos en más detalle en otro pasaje.

El tercer ejemplo de la lista de firmantes del Manifiesto contra "el autoritarismo" hecho público en abril de 2020 por la Fundación Internacional para la Libertad que quiero destacar es el de Mauricio Macri, ex Presidente de Argentina (2015-2019). En tono con las posturas de Sanguinetti y Uribe, durante su presidencia Macri tuvo cuidado en no dejar lugar a dudas sobre su etnocentrismo, clasismo y colonialismo con respecto a los sectores populares, los pueblos indígenas y sus derechos. Durante el Foro Económico de Davos de 2018, al cual asistió como Presidente temporal del G20, Macri hizo referencia a las negociaciones entonces en curso para concretar un Tratado de Libre Comercio entre el Mercado Común del Sur (MERCOSUR) y la Unión Europea y afirmó: “[y]o creo que la asociación entre el Mercosur y la Unión Europea es natural porque en Sudamérica todos somos descendientes de Europa" (Macri, 2018). Cabe recordar que el 9 de julio de 2016,

4 "Minga" es un concepto indígena referido a formas solidarias de acción colectiva. 
durante la celebración del Bicentenario de la Declaración de Independencia de Argentina, famosamente Macri se disculpó ante el Rey Emérito de España, quien lo acompañaba en la ceremonia, por la decisión de independizarse tomada en 1816 (Macri 2016), reafirmando su posición de subordinación colonial, algo que debe haber sorprendido al propio monarca emérito. Más allá de esta anécdota, lo que quiero enfatizar en conexión con el argumento de esta sección es la política de Macri en relación con las comunidades indígenas del país y los sectores populares en general. Durante la campaña electoral de 2015, Macri logró utilizar a algunas comunidades indígenas para fortalecer su mensaje, lo cual finalmente le dio resultados en la elección que lo convirtió en Presidente. El carácter utilitarista de dicha relación fue puesto de manifiesto en el título de un trabajo publicado durante la campaña: “[e]l día que Macri descubrió a los indígenas” (Balazote y Valverde, 2015; ver también: ENDEPA, 2015), en referencia al acercamiento de Macri con Félix Díaz, líder de la comunidad indígena QOM de la Provincia de Formosa, a quien luego nombraría a cargo del Consejo Consultivo y Participativo de los Pueblos Indígenas de la República Argentina. Hacia fines del período presidencial de Macri, el propio Díaz se encargaría de evaluar la trayectoria del gobierno en este tema: “[e]s una deuda que tuvo esta gestión con nosotros. Con la causa indígena, la actuación fue casi nula, se hicieron cosas mínimas en territorios, pero claramente no estamos conformes" (Diario La Mañana, 2019). Díaz ya se había manifestado previamente al respecto, por ejemplo en enero de 2018, en el marco de graves circunstancias que afectaban a diversas comunidades indígenas del país, Díaz declaró que "[t]eníamos expectativas cuando Macri nos visitaba, pero a las promesas se las llevó el viento. No es novedoso para nosotros que el gobierno no responda a nuestros pedidos [... estamos] muy preocupados por lo que está pasando a nivel nacional [...] mantengo diálogo con el gobierno pero no hay respuesta a nuestros planteos" (Servindi, 2018).

Esos comentarios de Díaz se dieron en un contexto generado por lo que un artículo publicado por el diario Financial Times del Reino Unido tituló "el impulso de Macri a las inversiones intensifica los conflictos por la tierra en Argentina", en el que se refirió a la existencia de más de 200 conflictos conectados con tierras indígenas en el país, que iban en camino a profundizarse debido a la política "pro-negocios" que estaba siendo implementada por Macri y que ya había producido sus primeras víctimas (Mander, 2017). Esto se daba porque, más allá de su utilización electoral de la problemática indígena, 
desde el inicio Macri había involucrado a su gobierno en acciones represivas contra los reclamos de las comunidades indígenas. Eso se hizo particularmente notorio en el ataque contra las comunidades mapuches de la Patagonia que continúan reclamando la devolución de sus territorios apropiados, dadas las enormes riquezas naturales allí existentes, muchos de los cuales desde la década de 1990 han sido entregados a empresarios, millonarios y empresas transnacionales. Un punto álgido de este proceso se dio en agosto de 2016 con la desaparición y posterior hallazgo del cuerpo sin vida del militante Santiago Maldonado, quien apoyaba una movilización mapuche en reclamo de sus tierras ancestrales que fue duramente reprimida por las fuerzas de la Gendarmería Nacional, un caso que aún sigue su proceso en la justicia y por el que altos cargos del gobierno de Macri han sido denunciados. Este hecho tuvo alta repercusión internacional, como lo puso de manifiesto un informe publicado en The Conversation, basado en Boston, Estados Unidos, titulado "[u]na muerte misteriosa en Argentina pone la atención sobre las luchas por tierras indígenas", que declaró: "la desaparición, las protestas y el controvertido descubrimiento [del cuerpo ] de Maldonado indican un aumento de la represión política durante el gobierno de Mauricio Macri, como lo muestra su tratamiento de los movimientos sociales y los grupos indígenas" (Clare, Mason-Deese et al., 2017). En conexión con esto, en una entrevista publicada por El Salto, de Madrid, la lideresa mapuche Moira Millán, de la Provincia de Chubut, reflexionando sobre el caso Maldonado y sus implicaciones, afirmó que

El pueblo mapuche es un obstáculo para ellos, estamos impidiendo que se desarrolle el extractivismo. Primero porque nuestra lógica no es capitalista, nosotros creemos que el valor sagrado es la vida, no la propiedad. Esas cosas no se venden, no tienen precio. No hay posibilidad de negociar con nosotros. Entonces, el único camino es eliminarnos, porque no pueden neutralizarnos mediante la cooptación. [...] El Gobierno de Macri ha presupuestado la muerte contra nosotros, se empeña en hacer una segunda Campaña del Desierto (Azcárate, 2017).

Debe recalcarse que la relativa notoriedad mediática alcanzada por la situación de las comunidades mapuches tiende a invisibilizar la situación de otras comunidades indígenas, como las de la región Noroeste de Argentina (NOA), una de las zonas históricamente más pobres y abandonadas del país. En conexión con esto, en enero de 2019 representantes de estas comunidades publicaron una llamada de "Alerta por incumplimiento y desamparo sistemático del gobierno de Macri a los Territorios Indígenas", en la cual denunciaron 
al Organismo de DDHH de la Nación, por la falta de garantías, intervención y pronunciamiento sobre los avasallamientos y violaciones de los Derechos Humanos que están ocurriendo en los Territorios Indígenas, con imputaciones, encarcelamientos, persecuciones, armado de causa, intento de homicidio, violencia síquica y física a Nuestras Autoridades Indígenas, traducidos en la "CRIMINALIZACION A LA DEFENSA LEGITIMA DE NUESTROS TERRITORIOS, del cual somos poseedores ancestrales como PUEBLOS PREEXISTENTES" como así también lo responsabilizamos ante cualquier daño que ocurriere con nuestra integridad física. [...] Las Organizaciones Indígenas del NOA, responsabilizamos al INAI [Instituto Nacional de Asuntos Indígenas], [Secretaría de] .DD.HH. [de la] Nación y [al] Presidente de la Nación Mauricio Macri, ante la vulneración de derecho y atropellos que se profundizan en nuestras Comunidades; como así también ante el abandono del Estado y desamparo de la Secretaria de Derechos Humanos y Pluralismo Cultural de la Nación, quien no brinda la asesoría legal, ni garantiza el acceso a la justicia que debería proveer el INAI, dejando a las 2.000 Comunidades Indígenas Preexistentes en una situación de inseguridad jurídica (sic) (Indymedia Argentina, 2019).

Ahora bien, el legado del ex Presidente Macri no se limitó a este abandono, discriminación y represión sistemática de las comunidades indígenas y de los sectores populares. Desplazado del poder en las elecciones presidenciales de 2019, se ha dedicado a atacar las políticas de protección de la salud pública implementadas por el gobierno del Presidente Alberto Fernández (2019-), que a pocos meses de iniciada su gestión en diciembre de 2019 se encontró con el inicio de la pandemia de COVID-19. Hablando el día 4 de marzo de 2020 en un evento internacional de la Fundación Libertad y Desarrollo, una entidad político-empresarial basada en Guatemala, el ex Presidente afirmó que "[m]ás importante que el Coronavirus es el populismo" (Macri, 2020), haciendo referencia al que el nuevo gobierno había abandonado las políticas neoliberales extremas que él había promovido y estaba implementando políticas urgentes de protección de la población, que han sido reconocidas internacionalmente por dar centralidad al cuidado de la vida de las personas y reducir significativamente la tasa de letalidad de la pandemia en Argentina (Goñi y Phillips, 2020; Sugarman, 2020). Macri incluso ha apoyado públicamente acciones de desobediencia civil contra las medidas de aislamiento y distancia social implementadas por el gobierno para frenar la circulación del coronavirus, acciones que incluyen ataques contra los médicos epidemiólogos que asesoran al Presidente Fernández y equiparan las medidas de protección de la salud con la Dictadura Militar que asoló al país entre 1976 y 1983: el gobierno habría instalado una "infectadura [que] es la máscara del totalitarismo" 
(Clarín, 2020; La Política on Line, 2020). Esta presión del ala más extrema del sector político liderado por Macri consiguió inducir al gobierno a acelerar el levantamiento de las medidas de aislamiento social, lo cual ha sido asociado con el rápido crecimiento en el número de infectados y fallecidos por COVID-19 durante agosto de 2020 (Rey, Calatrava et al., 2020).

Volviendo al Manifiesto liderado por Vargas Llosa, la participación de personas como Sanguinetti, Uribe y Macri, por dar solamente unos pocos ejemplos destacables de la lista de firmas, nos lleva a reflexionar sobre el significado de los conceptos de "libertad", "autoritarismo" y "solidaridad" que comparten las instituciones y personalidades promotoras y firmantes del texto, aunque claramente el tercer concepto no ocupa un lugar destacado en su vocabulario. El Manifiesto dedica críticas a las "dictaduras de Venezuela, Cuba y Nicaragua", así como a

“España y la Argentina [donde] dirigentes con un marcado sesgo
ideológico pretenden utilizar las duras circunstancias para acaparar
prerrogativas políticas y económicas que en otro contexto la ciudadanía
rechazaría resueltamente. En México arrecia la presión contra la empresa
privada y se utiliza el Grupo de Puebla para atacar a los gobiernos de
signo distinto" (Fundación Internacional para la Libertad (FIL) 2020).

Ni una mínima referencia al autoritarismo de Donald Trump, Presidente de los Estados Unidos (2017-) o de Jair Bolsonaro, Presidente de Brasil (2019-), para mencionar dos ejemplos extremos de autoritarismo vergonzosamente silenciados en el Manifiesto, a pesar de que ambos gobiernos han demostrado un desprecio singular por la vida humana, abiertamente promueven la violencia racial y política, se burlan de los principios básicos de la democracia y asocian la idea de "solidaridad" con el "comunismo" y el "autoritarismo". Tampoco hay referencia a Miguel Juan Sebastián Piñera Echenique, Presidente de Chile (2018-), a pesar de que en octubre de 2019 Amnesty International envió una comisión investigadora y urgió "nuevamente al Presidente Piñera poner fin a la violenta represión de aquellos que hoy ejercitan su derecho legítimo a la protesta pacífica" (Guevara-Rosas, 2019), represión que ha causado muchas víctimas inocentes y continúa siendo particularmente cruel con las comunidades indígenas y los sectores populares. De hecho, Piñera se ha beneficiado políticamente de la pandemia, ya que las medidas de control militarizado de la población que tomó le permitieron reducir las movilizaciones de protesta, a la vez que su implementación de políticas "populistas" de distribución de ayuda, similares a las implementadas por Argentina, le posibilitaron recuperarse de los 
bajos niveles de aprobación que su gobierno había tenido a fines de 2019 (Mander, 2020). Si bien en una entrevista que dio al diario chileno La Tercera Vargas Llosa hizo referencias a la mala gestión de la pandemia en Estados Unidos, el Reino Unido y Brasil (Retamal, 2020), eso no modifica la intencionalidad ni las implicaciones de ese Manifiesto publicado en abril de 2020.

\section{Conclusiones: otras solidaridades y otros órdenes sociales}

Si bien en la sección previa me concentré en enfatizar el ataque injustificado contra las formas de solidaridad institucionalizada que, con distintos resultados, se han implementado para proteger la vida de las poblaciones humanas contra la devastación causada por la pandemia, obviamente no es posible romantizar la solidaridad centrada en el Estado. Es un dato objetivo que las formas de protección coordinadas desde el Estado constituyen la única posibilidad de protección de la que disponen amplios sectores de las poblaciones humanas, evidentemente sobre todo los sectores más vulnerables, que no pueden acceder a los sistemas privados de salud. La crítica legítima contra el carácter autoritario, verticalista y no participativo, no sujeto al control democrático, que puede asumir la gestión pública de desastres como las pandemias, sin duda es una tarea permanente de la que no podemos abdicar en la lucha por la democratización substantiva de nuestras sociedades. Del mismo modo, no podemos abdicar de la crítica contra el uso histórico de la solidaridad institucionalizada como mecanismo de dominación y cooptación y como paliativo que permite a los sectores dominantes posponer indefinidamente las reformas estructurales que permitan transformar radicalmente, en el sentido clásico de transformarlo desde la raíz, el orden social dominante. Sin embargo, a pesar de esas y otras graves imperfecciones y de las implicaciones del carácter capitalista de estos procesos, la solidaridad institucionalizada continúa siendo en esta etapa histórica una de las formas más desarrolladas de protección de los derechos básicos y la única de la que disponen millones de personas, aunque solo accedan a esa protección de modo intermitente, precario, en el mejor de los casos. La actual pandemia nos recuerda con crudeza este dato objetivo, que nos confronta con una serie de contradicciones y caminos aparentemente sin salida. Para los sectores oprimidos y con conciencia de dicho estado de opresión, como es el caso de buena parte de las comunidades indígenas y 
afrodescendientes y de los sectores populares marginalizados y excluidos, es claramente contradictorio esperar que el Estado opresor pueda ser el proveedor de la protección requerida en las extremas circunstancias creadas por la pandemia. De hecho, en muchos casos, quienes controlan el Estado ni siquiera están interesados en garantizar el cumplimiento de esa función para dichos sectores, como ha quedado de manifiesto en diversos países.

En relación con esto, la necesidad de confrontar los efectos de la pandemia en ausencia de un Estado mínimamente protector, las comunidades indígenas y afrodescendientes han tomado un amplio rango de medidas de solidaridad "defensivas", intracomunitarias, que van desde el cierre de sus territorios al acceso por parte de personas externas a sus comunidades, como medida de aislamiento protector, hasta procesos de recuperación de conocimientos ancestrales relacionados, por ejemplo, con elementos naturales que sirven como desinfectantes o prácticas medicinales curativas, entre muchas otras iniciativas (OHCHR, 2020). También han desarrollado formas de solidaridad extracomunitarias, por ejemplo en el caso antes mencionado de las "mingas" solidarias organizadas por las comunidades indígenas del Departamento del Cauca en Colombia, para distribuir alimentos en las ciudades donde habita la población blanca y mestiza (CRIC, 2020), o las iniciativas del Movimento Sem Terra (MST) en Brasil, que distribuye buena parte de su producción de alimentos agroecológicos en diversas partes del país y también desarrolla actividades de medicina comunitaria en asociación con especialistas de otros países (Engelmann y Ferreira, 2020). Esto último contrasta con las actividades denunciadas por el propio MST y numerosas organizaciones sociales sobre la "farsa de las donaciones" realizadas por empresas privadas vinculadas a los agronegocios y otras actividades extractivistas que aprovechan la pandemia para autopublicitarse con la distribución de alimentos (Amigos da Terra Brasil et al., 2020).

Indudablemente, estas y otras formas de solidaridad que persisten y se reproducen a pesar del negacionismo y la discriminación dominantes son una manifestación también objetiva de los complejos y altamente diversos procesos de resistencia en defensa de la vida que pueden identificarse a escala planetaria. Como lo expresó con nitidez la antes citada lideresa mapuche Moira Millán, de la Provincia de Chubut, Argentina, en una entrevista que le hicieron en España, 
[c]reo en la solidaridad de los pueblos, en su capacidad de articularse, de tramar sueños. Y que pongamos por encima de todo ser guardianes de la vida. Vengo [a Europa] en busca de eso, de lazos solidarios para enfrentar a este enemigo común [el capitalismo extractivista]. Y tengo esperanza de que algo encontraré acá. Hay que pensar el arte de habitar de una manera muy diferente a como hemos venido viviendo durante estos siglos de imposición colonial. Generar una conciencia distinta que irá a parir una nueva sociedad que definirá el nuevo orden político, económico y social (Azcárate, 2017).

Este mensaje desde el corazón de nuestras sociedades indígenas contrasta con el ejemplo del Manifiesto "contra el autoritarismo" y de sus firmantes destacados, que ha sido adecuado para el análisis en este artículo. Ese análisis permite hacer observables con nitidez los fundamentos de la solidaridad que cohesiona a importantes sectores de las fracciones intelectuales, empresariales y políticas dominantes en América Latina. Desde una perspectiva, todo proceso de formación de solidaridades es un proceso de inclusiónexclusión, más allá de las pretensiones discursivas de universalidad que se puedan postular, legítimas o no. Pero ¿cuáles son los lazos de solidaridad social que integran a los representantes contemporáneos del darwinismo social y el colonialismo, que defienden la desigualdad estructural y hasta la violencia étnica y racista en nombre de la “civilización"? La pandemia ha permitido hacer observable lo que de alguna forma estos sectores han conseguido disimular en gran medida y que amplios sectores de la población han naturalizado. Queda por ver si, remitiéndonos a Jean Piaget (Piaget, 1977), la observabilidad de estos procesos, ya no solo en América Latina, contribuirá al desarrollo de niveles más elevados de toma de conciencia, que permitan avanzar en la desnaturalización de las desigualdades e injusticias estructurales y fundamenten el desarrollo de formas de solidaridad más integradoras, a nivel de la especie, que permitan generar la emergencia de órdenes sociales superadores, más justos y substantivamente democráticos. Inesperadamente, quizás, los impactos de la pandemia pueden abrir algunas oportunidades para que dichos procesos puedan prosperar.

\section{Referencias}

ADENCH - Asociación de Descendientes de la Nación Charrúa - Clan Choñik Hum Pampa y CONACHA - Consejo de la Nación Charrúa (2020). “Descendientes de Charrúas Refutan 
a Julio María Sanguinetti". Semanario Crónicas del Este. Maldonado, Uruguay. Disponible en: http://cronicasdeleste.com.uy/Noticias/descendientes-de-charruas.html. Consultado en agosto de 2020.

Amigos da Terra Brasil y otros (2020). “A farsa das doações no combate à Covid-19 nos setores de plantações de monoculturas de árvores, agronegócio, petróleo e mineração no Brasil". Disponible en: https://mst.org.br/2020/04/16/acoes-de-solidariedade-semterra-sem-espalham-pelo-pais-durante-pandemia/. Consultado en agosto de 2020.

Amira, R. (2016). "Uribe también saca la piedra a los afro". Colombia Plural. Disponible en: https://colombiaplural.com/racismo-d-uribe/. Consultado en agosto de 2020.

Azcárate, A. (2017). "El Gobierno de Macri ha presupuestado nuestra muerte". El Salto, Madrid. Disponible en: https://www.elsaltodiario.com/pueblos-originarios/mapucheargentina-moira-millan-gobierno-macri-maldonado-presupuestado-nuestra-muerte. Consultado en agosto de 2020.

Balazote, A. y S. Valverde (2015). “El día que Macri descubrió a los indígenas.” Disponible en: https://www.politicargentina.com/notas/201511/9506-el-dia-que-macridescubrio-a-los-indigenas.html. Consultado en agosto de 2020.

Baldwin, J. M. (1910). “The Basis of Social Solidarity.” American Journal of Sociology 15(6): 817-831.

Baldwin, P. (1990). The Politics of Social Solidarity. Class Bases of the European Welfare State 1875-1975. Cambridge: Cambridge University Press.

BBC News (2020). “Álvaro Uribe: Corte Suprema de Colombia ordena el arresto domiciliario del expresidente por 'riesgos de obstrucción a la justicia'." BBC News. Disponible en: https://www.bbc.com/mundo/noticias-america-latina-53604677. Consultado en agosto de 2020.

Clare, N., L. Mason-Deese, y V. Habermehl (2017). "A mysterious death in Argentina draws attention to indigenous land struggles." The Intercept. Disponible en: https://theconversation.com/a-mysterious-death-in-argentina-draws-attention-toindigenous-land-struggles-86028. Consultado en agosto de 2020.

Clarín (2020). Críticas al Gobierno "La democracia está en peligro", la carta abierta de intelectuales y científicos que alerta sobre el "eficaz relato de la infectadura". Disponible en: https://www.clarin.com/politica/-democracia-peligro-carta-abierta-intelectualescientificos-alerta-eficaz-relato-infectadura-_0_AxrZQ605F.html. Consultado en agosto de 2020.

Correa García, N. (2020). “Lo 'civilizado’ y nuestra memoria: sobre los dichos de Julio María Sanguinetti en relación con la matanza de Salsipuedes.” La Diaria. Disponible en: https://ladiaria.com.uy/opinion/articulo/2020/6/lo-civilizado-y-nuestra-memoriasobre-los-dichos-de-julio-maria-sanguinetti-en-relacion-con-la-matanza-desalsipuedes/. Consultado en agosto de 2020. 
Correia, M. y A. Dip (2020). "Profetas del caos: el poder de los negacionistas en Brasil", Agencia Pública, Brasil. Disponible en: https://ojo-publico.com/1865/profetas-del-caosel-poder-de-los-negacionistas-en-brasil. Consultado en agosto de 2020.

CRIC - Consejo Regional Indígena del Cauca (2019). "La minga por la defensa del territorio, la democracia, la vida, la justicia y la paz." Disponible en: https://www.criccolombia.org/portal/la-minga-por-la-defensa-del-territorio-la-democracia-la-vida-lajusticia-y-la-paz/. Consultado en agosto de 2020.

CRIC - Consejo Regional Indígena del Cauca. (2020). "La Minga de la comida CRICEjemplo de solidaridad.” Disponible en: https://www.cric-colombia.org/portal/laminga-de-la-comida-cric-ejemplo-de-solidaridad/. Consultado en agosto de 2020.

Crosby, A. W. (1988). Imperialismo ecológico. La expansión biológica de Europa, 9001900. Barcelona: Editorial Crítica.

Delgado Cultelli, M. (2016). "La Reacción Colonialista a la Reemergencia Indígena." Correo Montevideo, Foro Batllista. Disponible en:

http://www.correodelosviernes.com.uy/La-Reaccion-Colonialista-a-la-ReemergenciaIndigena..asp. Consultado en agosto de 2020.

Diario La Mañana (2019). “Para Félix Díaz, el gobierno de Macri 'mostró falencias' con los pueblos indígenas y ‘dejó deudas' en materia territorial”. Diario La Mañana. Ciudad de Formosa. Disponible en: https://www.xn--lamaanaonline-

lkb.com.ar/noticia/34666/para-flix-daz-el-gobierno-de-macri-mostr-falencias-con-lospueblos-indgenas-y-dej-deudas-en-materia-territorial/. Consultado en agosto de 2020.

CEPAL - Comisión Económica para América Latina y el Caribe y ACNUDH - Oficina del Alto Comisionado de las Naciones Unidas para los Derechos Humanos (2020). "Afrodescendientes en América Latina y el Caribe. Elaboración de indicadores para medir y contrarrestar las desigualdades". Santiago de Chile: CEPAL-ACNUDH.

CEPAL - Comisión Económica para América Latina y el Caribe y FILAC - Fondo para el Desarrollo de los Pueblos Indígenas de América Latina y el Caribe (2020). "Los pueblos indígenas de América Latina - Abya Yala y la Agenda 2030 para el Desarrollo Sostenible. Tensiones y desafíos desde una perspectiva territorial". Santiago de Chile: CEPAL-FILAC.

Elias, N. (1994). The Civilizing Process. The History of Manners, and State Formation and Civilization. Oxford y Cambridge, MA: Basil Blackwell.

ENDEPA - Equipo Nacional de Pastoral Aborigen (2015). "Documento de los Pueblos Indígenas presentado al Presidente Macri.” Buenos Aires. Disponible en:

https://www.endepa.org.ar/documento-de-los-pueblos-indigenas-presentado-alpresidente-macri/. Consultado en agosto de 2020.

Engelmann, S. y J. Ferreira (2020). “Ações de solidariedade Sem Terra se espalham pelo país durante pandemia." Disponible en: https://mst.org.br/2020/04/16/acoes-desolidariedade-sem-terra-sem-espalham-pelo-pais-durante-pandemia/. Consultado en agosto de 2020. 
FIL - Fundación Internacional para la Libertad. (2020). “Que la pandemia no sea un pretexto para el autoritarismo." Disponible en: https://fundacionfil.org/manifiesto-fil/. Consultado en agosto de 2020.

Goñi, U. y T. Phillips (2020). “You can't recover from death': Argentina's Covid-19 response the opposite of Brazil's." The Guardian. Disponible en:

https://www.theguardian.com/world/2020/may/10/argentina-covid19-brazilresponse-bolsonaro-fernandez. Consultado en agosto de 2020.

González Casanova, P. (1963). “México: Desarrollo y Subdesarrollo.” Desarrollo Económico 3(1/2): 285-302.

Guevara-Rosas, E. (2019). “Chile: Amnesty International announces research mission to document grave human rights violations". Ciudad de México: Amnesty International. Disponible en: https://www.amnesty.org/en/latest/news/2019/10/chileinvestigacion-para-documentar-violaciones-derechos-humanos/. Consultado en agosto de 2020.

Hobsbawm, E. (1975). The Age of Revolution. 1789-1848. Londres: Weidenfeld \& Nicolson.

Indymedia Argentina (2019) "Alerta por incumplimiento y desamparo sistemático del gobierno de Macri a los Territorios Indígenas.” Disponible en:

https://argentina.indymedia.org/2019/01/02/alerta-por-incumplimiento-ydesamparo-sistematico-del-gobierno-de-macri-a-los-territorios-indigenas/. Consultado en agosto de 2020.

La Política on Line (2020). Protests in Argentina against the lockdown. La Política on Line. Disponible en: https://www.lapoliticaonline.com/nota/127905-protests-inargentina-against-the-lockdown/. Consultado en agosto de 2020.

Lissardy, G. (2020). “Los evangélicos y el coronavirus: los grupos religiosos que resisten las restricciones contra el covid-19 en algunos países de América." BBC News. Disponible en: https://www.bbc.com/mundo/noticias-internacional-52612458. Consultado en agosto de 2020.

Macri, M. (2016). "Discurso Presidencial por el Bicentenario de la Declaración de la Independencia.” Tucumán, Presidencia de la Nación, República Argentina. Disponible en: https://youtu.be/TVjQla7 OPY. Consultado en agosto de 2020.

Macri, M. (2018). "Special Address by Mauricio Macri, President of Argentina and Chair of the G20, on the 2018 G20 Agenda." Davos, World Economic Forum. Disponible en: https://www.weforum.org/events/world-economic-forum-annual-meeting2018/sessions/special-address-by-mauricio-macri-president-of-argentina-and-chair-ofthe-g20-on-the-2018-g20-agenda. Consultado en agosto de 2020.

Macri, M. (2020). “Más importante que el Coronavirus es el populismo.” Disponible en: https://youtu.be/ioS4tAGjvfE. V Encuentro Ciudadano: Centroamérica, amenazas y oportunidades compartidas; un destino común. Guatemala, Fundación Libertad y Desarrollo. Consultado en agosto de 2020. 
Mander, B. (2017). "Macri investment push intensifies Argentina's land conflicts." Financial Times. Disponible en: https://www.ft.com/content/fe5d3812-a7a0-11e7ab55-27219df83c97. Consultado en agosto de 2020.

Mander, B. (2020). “Chile's coronavirus outbreak helps revive Piñera's fortunes.” Financial Times. Disponible en: https://www.ft.com/content/7578b8a0-5fa8-4aca89e5-fd0bf2e78e3a. Consultado en agosto de 2020.

Medina Gallego, C. (2020). "El asesinato de líderes sociales: la otra pandemia." Razón Pública. Bogotá. Disponible en: https://razonpublica.com/asesinato-lideres-sociales-laotra-pandemia/. Consultado en agosto de 2020.

Noticias Uno Colombia (2019). "Controversia en redes sociales por trinos del senador Álvaro Uribe”, Noticias Uno Colombia. Disponible en: https://youtu.be/XXovvaEY0Bg. Consultado en agosto de 2020.

OHCHR - Oficina del Alto Comisionado de las Naciones Unidas para los Derechos Humanos (2020). “Guía: COVID-19 y los derechos de los pueblos indígenas.” Ginebra: OHCHR. Disponible en:

https://www.ohchr.org/Documents/Issues/IPeoples/OHCHRGuidance COVID19 Indige nouspeoplesRights ES.pdf. Consultado en agosto de 2020.

OIT - Organización Internacional del Trabajo (1989). “C169 - Convenio sobre pueblos indígenas y tribales, 1989 (núm. 169).” Ginebra: OIT. Disponible en:

https://www.ilo.org/dyn/normlex/es/f?p=NORMLEXPUB:12100:0::NO::P12100_ILO_C ODE:C169. Consultado en agosto de 2020.

OPS - Organización Panamericana de la Salud (2020). “Consideraciones relativas a los pueblos indígenas, afrodescendientes y otros grupos étnicos durante la pandemia de la COVID-19." Washington, DC,: OPS. Disponible en:

https://www.paho.org/es/file/66327/download?token= CvHO7iU. Consultado en agosto de 2020.

Oualalou, L. (2019). “Los evangélicos y el hermano Bolsonaro.” Nueva Sociedad(280): 68-77.

Pachico, E., G. Sánchez-Garzoli y A. Isacson (2019). “What's at Stake When Uribe Testifies in Historic Colombia Case." Washington DC, Advocacy for Human Rights in the Americas (WOLA). Disponible en: https://www.wola.org/analysis/colombia-former-presidenturibe-testifies/. Consultado en agosto de 2020.

Pardo, D. (2020). "Masacre de Samaniego: una nueva matanza de jóvenes deja en evidencia el recrudecimiento de la violencia en Colombia." BBC News. Disponible en: https://www.bbc.com/mundo/noticias-america-latina-53801935. Consultado en agosto de 2020.

PARES - Fundación Fundación Paz y Reconciliación (2019). “Minga le responde a Uribe por 'promover el asesinato'." Bogotá: PARES. Disponible en:

https://pares.com.co/2019/04/08/minga-le-responde-a-uribe-por-promover-elasesinato/. 
Piaget, J. (1977). The Grasp of Consciousness. Londres: Routledge y Kegan Paul.

Poder Legislativo, República Oriental del Uruguay (2009). “Ley № 18.589. Día de la Nación Charrúa y de la Identidad Indígena." Montevideo, Poder Legislativo, República Oriental del Uruguay. Disponible en:

https://legislativo.parlamento.gub.uy/temporales/leytemp9015101.htm. Consultado en agosto de 2020.

Retamal, F. (2020). "Vargas Llosa critica a Trump, Bolsonaro y Johnson: 'Procedieron de forma irresponsable, pensando que podían sortear la amenaza'”. La Tercera. Santiago de Chile. Disponible en: https://www.latercera.com/culto/2020/04/13/vargas-llosacritica-a-trump-bolsonaro-y-johnson-procedieron-de-forma-irresponsable-pensandoque-podian-sortear-la-amenaza/. Consultado en agosto de 2020.

Reuters (2020). U.S. vice president calls for Colombia's Uribe to be released from house arrest. Disponible en: https://www.reuters.com/article/us-colombia-usa/u-s-vicepresident-calls-for-colombias-uribe-to-be-released-from-house-arrest-idUSKCN25B006. Consultado en agosto de 2020.

Rey, D., A. Calatrava y C. Torchia (2020). "Nerves fray in Argentina, where some flout pandemic lockdown." The Washington Post. Disponible en:

https://www.washingtonpost.com/world/the americas/nerves-fray-in-argentinawhere-some-flout-pandemic-lockdown/2020/08/12/ff161f2a-dcc3-11ea-b4f125b762cdbbf4 story.html. Consultado en agosto de 2020.

Sanguinetti, J. M. (2009). “El Charruismo.” El País. Montevideo. Disponible en: http://historico.elpais.com.uy/09/04/19/predit 411886.asp. Consultado en agosto de 2020.

Sanguinetti, J. M. (2016). “Charruismo estatal.” Correo de los Viernes. Montevideo, Foro Batllista. Disponible en: http://www.correodelosviernes.com.uy/Charruismoestatal.asp. Consultado en agosto de 2020.

Sanguinetti, J. M. (2020). “Sobre Rivera y Salsipuedes.” Montevideo. Disponible en: https://www.youtube.com/watch?v=FuClSM1lUc0. Consultado en agosto de 2020.

Servindi - Servicios de Comunicación Intercultural (2018). “Argentina: 'Teníamos expectativas con Macri... a las promesas se las llevó el viento'." Servindi - Servicios de Comunicación Intercultural Disponible en: https://www.servindi.org/actualidadnoticias/08/01/2018/teniamos-expectativas-con-macri-pero-las-promesas-se-lasllevo-el. Consultado en agosto de 2020.

Spencer, H. ([1850] 1883). Social Statics, or, The Conditions Essential to Human Happiness Specified. Nueva York: D. Appleton \& Co.

Sugarman, J. (2020). “Argentina Is Showing the World What a Humane Covid-19 Response Looks Like." The Nation. Disponible en: https://www.thenation.com/article/politics/coronavirus-argentina-humane-responseto-covid-19-look-like/. Consultado en agosto de 2020. 
Verdesio, G. (2014). "Un fantasma recorre el Uruguay: la reemergencia charrúa en un 'país sin indios'." Cuadernos de Literatura XVIII(36): 86-107.

Wallach, L. (2009). "Bush Grant of Medal of Freedom to Colombian President Uribe Is an Outrage Given his Government's Links with Right-Wing Paramilitaries and the Growing Attacks on Colombian Unionists, Afro-Colombian and Indigenous People", Public Citizen. Disponible en: https://citizen.typepad.com/eyesontrade/2009/01/bush-grant-ofmedal-of-freedom-to-colombian-president-uribe-is-an-outrage.html. Consultado en agosto de 2020 .

WOLA - Advocacy for Human Rights in the Americas (2020). "Ongoing Legal Process Against Uribe an Important Advance for Justice and Accountability in Colombia." Washington DC: WOLA. 